\title{
Reproduction De Heterotis Niloticus (Cuvier, 1829) De La Riviere Agneby (Cote d'Ivoire)
}

\author{
Kouakou Fokouo Kessia Irène \\ Koné Tiéhoua \\ Soro Yaya \\ N'Da Konan
}

Laboratoire de Biologie et Cytologie Animales, UFR des sciences de la nature, Université Nangui Abrogoua, Côte d'Ivoire

\section{Ouattara Mamadou}

Laboratoire d'Environnement et Biologie Aquatique,

UFR des sciences et gestion de l'environnement,

Université Nangui Abrogoua, Côte d’Ivoire

doi: 10.19044/esj.2016.v12n6p83 URL:http://dx.doi.org/10.19044/esj.2016.v12n6p83

\begin{abstract}
The reproductive biology of Heterotis niloticus has been studied from monthly samples taken from artisanal fishing on the Agneby river during the period june 2013 to May 2015. The whole sample consisted of 498 specimens of which 269 females and 229 males were examined. Sex-ratio observed was in favour of females $\left(1: 1.17 ; X^{2}=3.21 ; p>0.05\right)$. The monthly evolution of the gonado-somatic index (GSI), the liver-somatic index (LSI), the condition factor (K) and maturation stages suggests that this fish has two periods of reproduction. The two periods were respectively from june to august and from november to december. The standard length at first sexual maturity was $490.6 \mathrm{~mm}$ for females and $436.3 \mathrm{~mm}$ for males. The absolute fecundity was ranged from 5424 to 25591 oocytes in females of 595 $\mathrm{mm}$ to $780 \mathrm{~mm}$ standard length. The relative fecundity was $3752 \pm 1491$ oocytes/kg of female.
\end{abstract}

Keywords: Heterotis niloticus, Reproduction, fecundity, Agneby river

\section{Résumé}

La reproduction de Heterotis niloticus a été étudiée de juin 2013 à mai 2015 à partir des échantillons provenant de la pêche artisanale de la rivière Agnéby. Au total 498 spécimens dont 269 femelles et 229 mâles ont été examinés. La sex-ratio est en faveur des femelles $\left(1: 1,17 ; X^{2}=3,21 ; p>\right.$ $0,05)$. Le suivi mensuel de l'évolution du rapport gonado-somatique (RGS), 
du rapport hépato-somatique (RHS), du facteur de condition (K) et des stades de maturité révèle que ce poisson a deux périodes de reproduction. La première période de ponte se situe de juin à août tandis que la seconde intervient de novembre à décembre. Les tailles de première maturité sexuelle sont de 490,6 $\mathrm{mm}$ et 436,3 $\mathrm{mm}$ de longueur standard respectivement chez les femelles et les mâles. La fécondité absolue varie de 5424 à 25591 ovocytes chez des femelles de $595 \mathrm{~mm}$ à $780 \mathrm{~mm}$ de longueur standard avec une fécondité relative de 3752 1491 ovocytes/kg de poids corporel de femelle.

Mots-clés: Heterotis niloticus, Reproduction, Fécondité, Rivière Agnéby

\section{Introduction}

La reproduction constitue une étape essentielle dans le cycle de vie d'un poisson. L'équilibre démographique d'une espèce de poisson est étroitement lié au bon déroulement de cette phase (Parkinson et al., 1999).

Heterotis niloticus est un poisson de la famille des Osteoglossidae. Cette espèce a un fort potentiel aquacole en raison de sa forte croissance, sa double respiration, la bonne qualité gustative de sa chair et sa relative haute valeur commerciale (Monencham, 2009). Elle a été introduite en Côte d'Ivoire dans les lacs de barrage d'Ayamé, de Kossou, de Buyo et de Taabo (Gourène et Teugels, 1995). Dans la rivière Agnéby, elle est exploitée de façon régulière par la pêcherie artisanale. Cependant, aucune étude n'existe sur la biologie de sa reproduction dans ce milieu. En effet, les données portant sur les paramètres de la reproduction de $H$. niloticus sont celles étudiée en pisciculture par Reizer (1964), Micha (1973) et Monentcham (2009) respectivement en Côte d’Ivoire , en République Démocratique du Congo et au Cameroun. Les premières observations en milieu naturel sont celles de Moreau (1982) en Côte d'Ivoire, Moreau et Moreau (1982) à Madagascar et Adite et al. (2006) au Benin.

En outre, pour une gestion rationnelle des stocks ichtyologiques, il est nécessaire de disposer de connaissances sur les paramètres de la reproduction des espèces de poisson notamment, la taille à la première maturité sexuelle, la fécondité, la période de reproduction (Kao et al., 1999; Offem et al., 2009). L’objectif visé dans ce travail est de déterminer les paramètres de reproduction de $H$. niloticus dans la rivière Agnéby à travers la connaissance de sa sex ratio, de sa taille de première maturité sexuelle, de sa période de reproduction et de sa fécondité.

\section{Milieu, matériel et méthodes Milieu d'étude}

La rivière Agnéby est située entre $5^{\circ} 10$ et $6^{\circ} 08$ de latitude nord et ente $3^{\circ} 50$ et $4^{\circ} 41$ de longitude ouest (Goula et al., 2009). Elle s’écoule du 
nord au sud et prend sa source à Agoua dans le département de Bongouanou à l'altitude 250m (Girard et al., 1971). Ses affluents sont le M’pébo, le Kavi et le Séguié (Goula et al., 2009). Cette rivière traverse la région d'Agboville et se jette dans la lagune Ebrié près de Dabou et a un débit moyen annuel de $22 \mathrm{~m}^{3} / \mathrm{s}$ (Diomandé et al., 2009).

Son bassin versant couvre une superficie d'environ $8500 \mathrm{~km}^{2}$ et développe son lit sur environ $217 \mathrm{~km}$. La pente du bassin, de l'ordre de $1,25 \mathrm{~m} / \mathrm{km}$, est assez régulière et relativement faible. Une grande partie du bassin se trouve en dessous de $100 \mathrm{~m}$ avec une altitude moyenne de $105 \mathrm{~m}$ (Goula et al., 2009). Plus de la moitié du bassin versant de cette rivière est occupée par des plantations de caféiers, de cacaoyers, de bananiers et de palmiers à huile. Il existe également sur son cours de nombreux barrages à vocation agropastorale et pour l'alimentation en eau potable (Diomandé et al., 2009). Ce bassin se trouve dans une zone de climat équatorial humide à quatre saisons. La grande saison des pluies s'étend d'avril à juillet et la petite saison des pluies d'octobre à novembre. Ces deux saisons sont entrecoupées par la petite saison sèche d'août à septembre. La grande saison sèche couvre de décembre à mars (Bony, 2007).

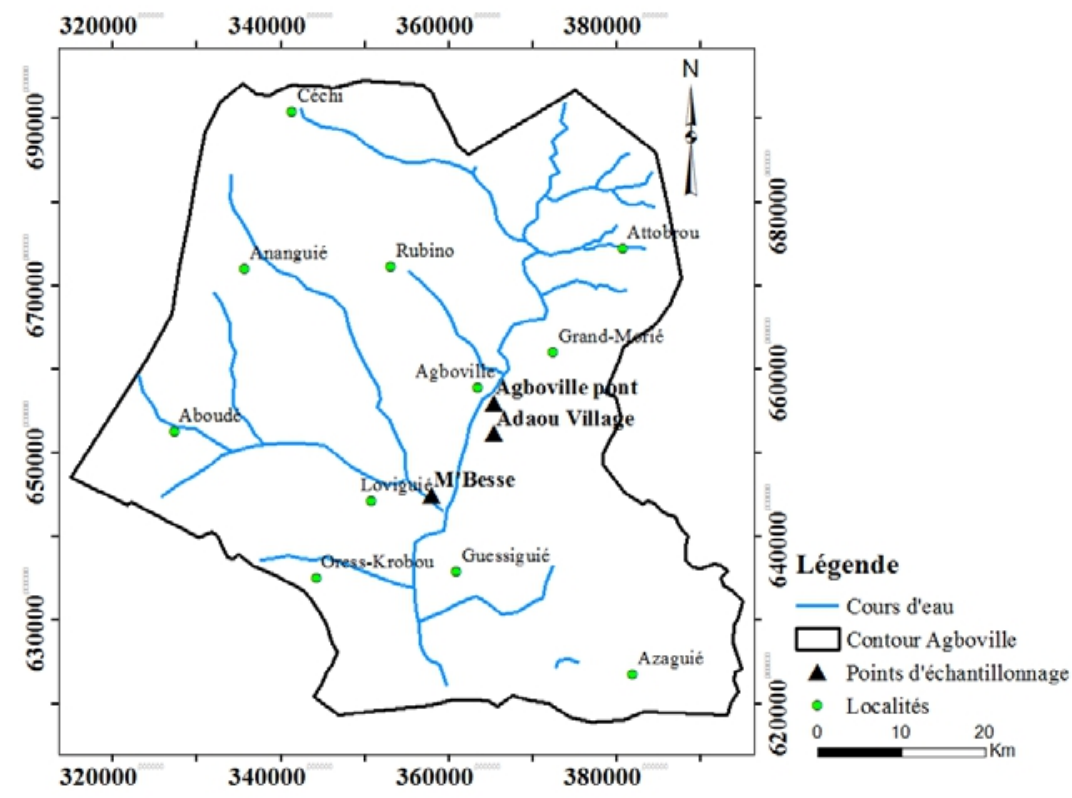

Figure 1: Présentation du milieu d’étude

\section{Matériel et méthodes}

Un échantillonnage mensuel à partir des débarquements de la pêche artisanale commerciale a été réalisé de juin 2013 à Mai 2015. Les engins de 
captures sont composés de filets éperviers de dimension de mailles variant de $10 \mathrm{~mm}$ à $30 \mathrm{~mm}$. La pêche s'effectue de jour à l'aide de pirogues monoxyles.

Sur chaque spécimen, la longueur standard et la longueur totale ont été relevées au millimètre près et le poids total et le poids éviscéré au gramme près. Chaque individu a ensuite été disséqué. Le sexe et le stade de maturité sexuel ont été déterminés selon l'échelle macroscopique de Moreau (1982). Les gonades et les foies ont ensuite été prélevés et pesés au gramme près.

La sex-ratio (M/F) exprimant la proportion relative des individus des deux sexes dans une population à un moment donné a été déterminée en utilisant la formle suivante:

$\mathrm{SR}=\mathrm{M} / \mathrm{F}$ avec $\mathrm{M}$ : mâles et $\mathrm{F}$ : femelles

Pour établir le cycle sexuel et déterminer la période de ponte, le rapport gonado-somatique (RGS) et le rapport hépato-somatique (RHS) ont été calculés mensuellement selon les formules suivantes.

$\mathrm{RGS}=(\mathrm{Pg} / \mathrm{Pe}) \times 100$ et $\mathrm{RHS}=(\mathrm{Pf} / \mathrm{Pe}) \times 100$

avec Pg: poids des gonades en grammes ; Pf: poids du foie en grammes ; Pe : poids éviscéré du poisson en grammes.

Le facteur de condition (K) a été également calculé en utilisant la formule :

$$
\mathrm{K}=\left(\mathrm{PV} / \mathrm{Ls}^{3}\right) \times 100
$$

avec $\mathrm{Pv}$ : poids vif du poisson en grammes et Ls : longueur standard du poisson en $\mathrm{cm}$.

Les fécondités absolue (nombre d'ovocytes dans un ovaire) et relative (nombre d’ovocyte par unité de poids corporel) ont été évaluées pour déterminer le potentiel reproducteur.

Le test de $\mathrm{khi}^{2}\left(\mathrm{X}^{2}\right)$ a été utilisé pour comparer les pourcentages des sex-ratio des différents mois et saisons à la sex-ratio théorique (1/1).

L'estimation de la taille de première maturité sexuelle $\left(\mathrm{Ls}_{50}\right)$ a été établie en ajustant par la fonction logistique d’une régression non linéaire, les pourcentages des individus matures par intervalle de classe de taille (Konan et al., 2013).

$$
P=\frac{1}{1+e^{-(\alpha+\beta L s)}} \times 100 \quad \text { avec } \quad L s_{50}=-\frac{\alpha}{\beta}
$$

$\mathrm{P}$ : proportions d'individus matures ; Ls : longueur standard (mm); $\alpha$ et $\beta$ : constantes

Les tailles de première maturité ont été également comparées en fonction du sexe. 


\section{Résultats}

\section{Sex-ratio}

Sur un ensemble de 498 spécimens récoltés, 269 femelles et 229 mâles ont été identifiés. La sex-ratio (mâle : femelle) de l'ensemble de l'échantillon correspond à $1: 1,17$ (Tableau 1). Cette différence n'est pas significative $\left(X^{2}=3,21 ; p>0,05\right)$. La variation mensuelle indique que la sex-ratio reste en faveur des femelles sauf les mois de juin 2013, juin 2014, juillet 2014, novembre 2014. Cependant, cette différence n'est significative qu'en juillet $2014\left(X^{2}=14,34 ; p<0,05\right)$. Quant à la variation saisonnière de la sexe ratio, elle est en faveur des mâles pendant la saison pluvieuse tandis que pendant la saison sèche elle est en faveur des femelles $\left(X^{2}=16,76\right.$; $p<$ $0,05)$

Tableau 1 : Variations mensuelle et saisonnière de la proportion des sexes et de la sex-ratio chez $H$. niloticus capturé dans la rivière Agnéby. ${ }^{*}$ différence significative (test $\mathrm{X}^{2}$ au seuil de $5 \%)$.

\begin{tabular}{cccccc}
\hline Mois & $\begin{array}{c}\text { Nombre de } \\
\text { Femelles }\end{array}$ & $\begin{array}{c}\text { Nombre de } \\
\text { Mâles }\end{array}$ & Total & $\begin{array}{c}\text { Sex-ratio } \\
\text { (M:F) }\end{array}$ & $\mathrm{X}^{2}$ \\
\hline Juin 2013 & 19 & 23 & 42 & $1: 0,82$ & 0,38 \\
Juillet & 16 & 11 & 27 & $1: 1,45$ & 0,93 \\
Août & 10 & 4 & 14 & $1: 2,5$ & 2,57 \\
Septembre & 7 & 3 & 10 & $1: 2,33$ & 1,6 \\
Octobre & 7 & 5 & 12 & $1: 1,4$ & 0,33 \\
Novembre & 11 & 7 & 18 & $1: 1,57$ & 0,89 \\
Décembre & 8 & 6 & 14 & $1: 1,33$ & 0,29 \\
Janvier 2014 & 3 & 3 & 6 & $1: 1$ & 0,00 \\
Février & 4 & 2 & 6 & $1: 2$ & 0,67 \\
Mars & 9 & 5 & 14 & $1: 1,8$ & 1,14 \\
Avril & 7 & 5 & 12 & $1: 1,4$ & 0,33 \\
Mai & 15 & 9 & 24 & $1: 1,66$ & 1,50 \\
Juin & 24 & 26 & 50 & $1: 0,92$ & 1,08 \\
Juillet & 18 & 49 & 67 & $1: 0,36$ & $14,34^{*}$ \\
Août & 12 & 7 & 19 & $1: 1,71$ & 1,32 \\
Septembre & 14 & 8 & 22 & $1: 1,75$ & 1,64 \\
Octobre & 12 & 11 & 23 & $1: 1,09$ & 0,04 \\
Novembre & 10 & 13 & 23 & $1: 0.76$ & 0,39 \\
Décembre & 17 & 11 & 28 & $1: 1,54$ & 1,29 \\
Janvier 2015 & 12 & 3 & 15 & $1: 4$ & $5,4^{*}$ \\
Février & 3 & 3 & 6 & $1: 1$ & 0,00 \\
Mars & 15 & 5 & 13 & $1: 3$ & $5,00^{*}$ \\
Avril & 8 & 2 & 5 & $1: 4$ & 3,6 \\
Mai & 8 & 8 & 12 & $1: 1$ & 0,00 \\
Total & 269 & 229 & 498 & $1: 1,17$ & 3,21 \\
\hline Saison pluvieuse & 155 & 169 & 324 & $1: 0,91$ & 0,60 \\
Saison Sèche & 114 & 60 & 174 & $1: 1,9$ & $16,76^{*}$ \\
\hline
\end{tabular}




\section{Variation mensuelle des stades de maturité sexuelle}

L'analyse de l'évolution de la maturité sexuelle chez les femelles met en évidence les stades II sur toute la période d'échantillonnage. Les femelles de stade I n’ont pas été observées durant les mois de septembre 2013 à décembre 2013 et d'octobre 2014 à novembre 2014. Quant aux femelles de stade III, elles sont absentes durant les mois de septembre, janvier et février pour les deux années. Les individus de stade IV apparaissent de juin à juillet et de novembre à décembre 2013, de mai à juillet et d'octobre à novembre 2014. Ils sont enfin présent en mai 2015. Les femelles du stade V sont présentes de juin à août et de novembre à décembre 2013, de juin à août et d'octobre à décembre 2014. Enfin, le stade VI apparaît de juin à août et en décembre pour les deux années (Figure 2).

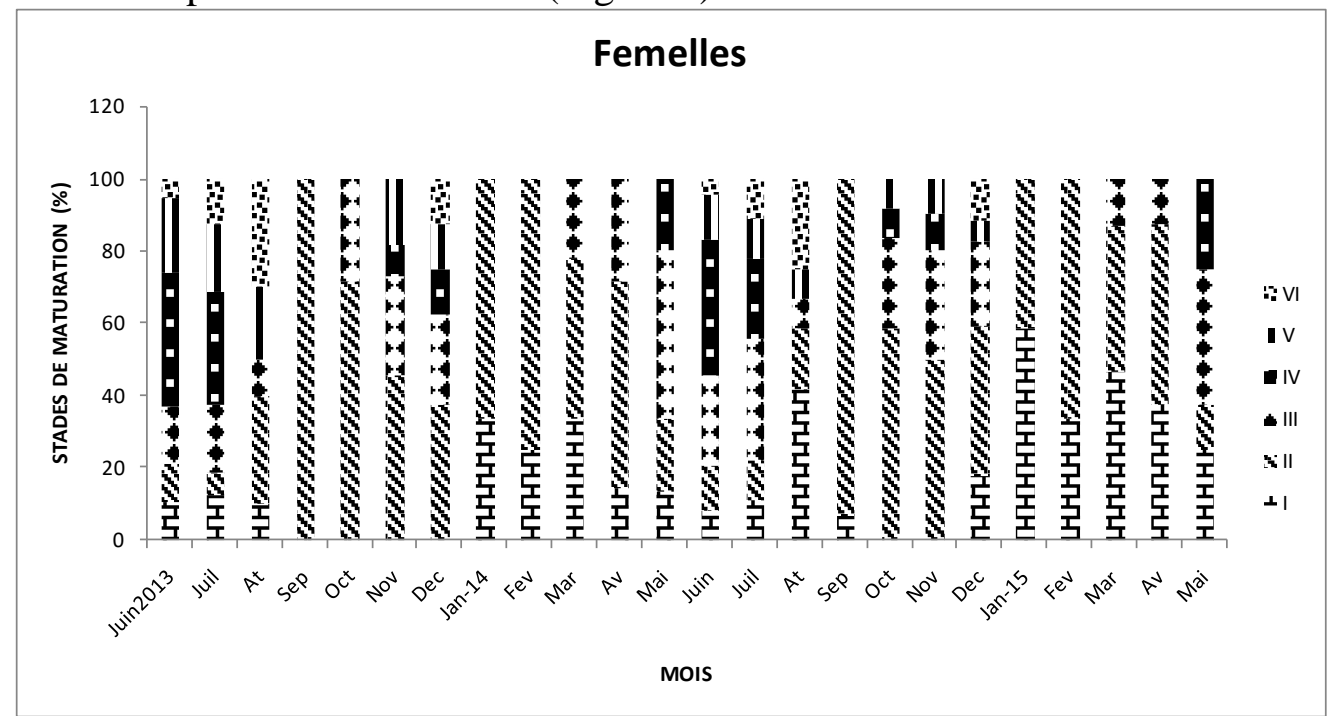

Figure 2 : Evolution mensuelle des proportions des différents stades de maturation sexuelle des femelles de Heterotis niloticus de la rivière Agnéby de juin 2013 à mai 2015.

\section{Rapport gonado-somatique (RGS) et période de reproduction}

L'examen de la courbe de variation mensuelle du RGS pendant les années d'étude montre chez les femelles une évolution similaire d'une année à l'autre (Figure 3).

Les plus faibles valeurs de RGS sont observées de janvier à avril puis d'août, à octobre. A partir du mois de mai, le RGS croît pour atteindre le pic en juin $(2,03 \pm 1,33$ et $2,77 \pm 1,27)$ respectivement en 2013 et 2014. Ensuite, un pic plus faible que le premier est atteint en novembre $(0,94 \pm 1,41$ et 0,86 $\pm 1,05)$ en 2013 et 2014 respectivement.

Chez les mâles, la plus forte valeur du RGS moyen est observée en juin $(0,11 \pm 0,03$ et $0,13 \pm 0,07)$ respectivement en 2013 et 2014 . Un plateau 
est enregistré en octobre $2013(0,09 \pm 0,04)$, puis un léger pic est observé en novembre $2014(0,08 \pm 0,01)$.

Ces variations mensuelles revèlent que cette espèce se reproduit deux fois dans l'année. La première période de reproduction a lieu de juin en août et la seconde de novembre à décembre.
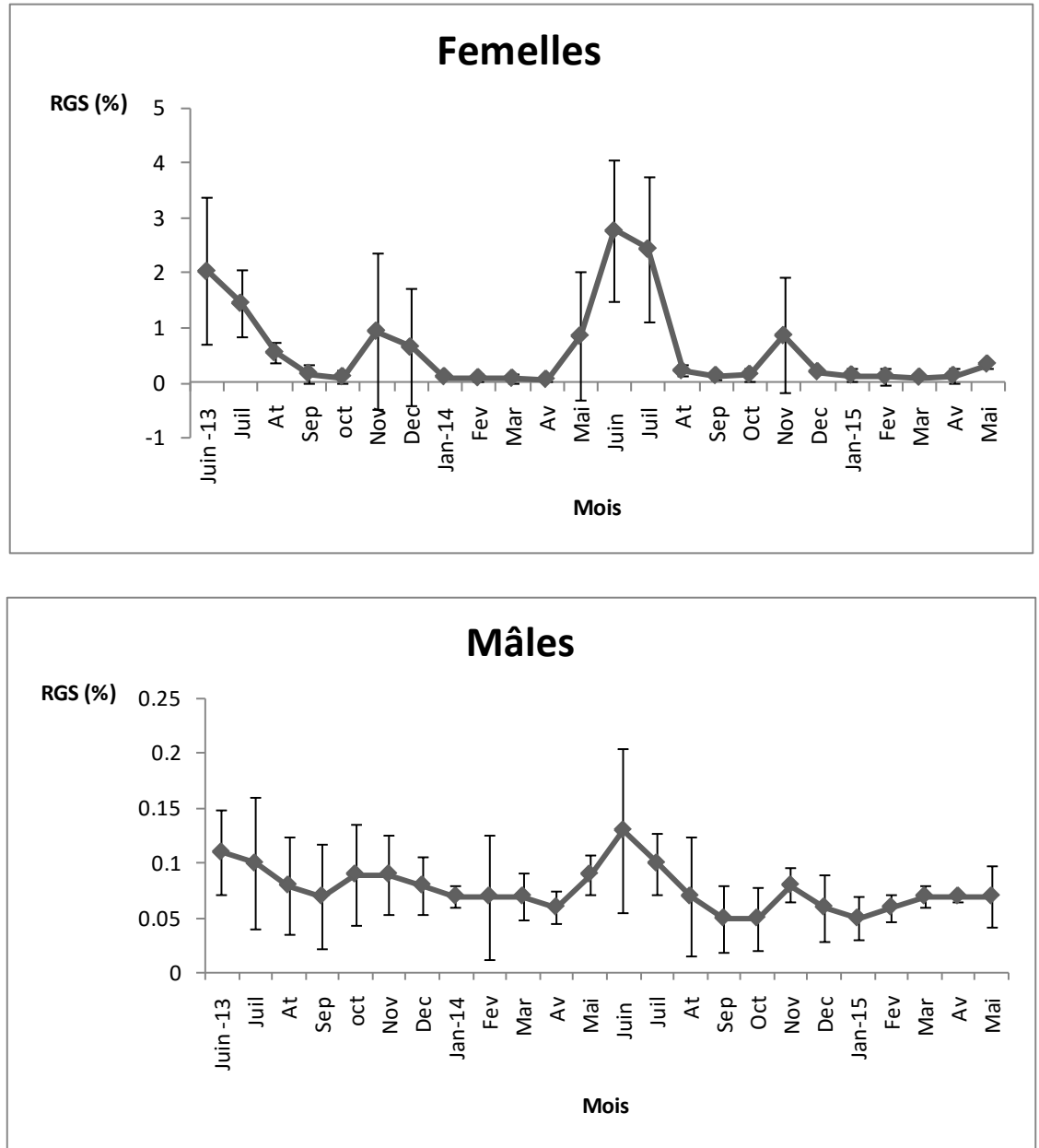

Figure 3 : Variations mensuelles du rapport gonado-somatique (RGS) des femelles et des mâles de Heterotis niloticus de la rivière Agnéby de juin 2013 à mai 2015.

\section{Rapport Hépato-somatique}

Les courbes d’évolution mensuelle du RHS moyen chez les deux sexes sont similaires à celles du RGS (Figure 4). Chez les femelles, le pic du

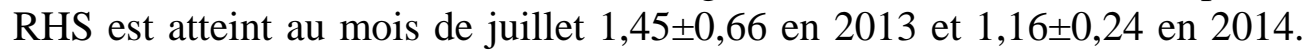
Le RHS baisse ensuite lentement pour atteindre le minimum en Février $(0,81 \pm 0,17$ et $0,75 \pm 0,16)$ respectivement en 2014 et 2015. Concernant le RHS mensuel des mâles, les valeurs les plus élevées sont observées en juin $2013(1,13 \pm 0,26)$ et en juillet $2014(1,39 \pm 0,37)$. 

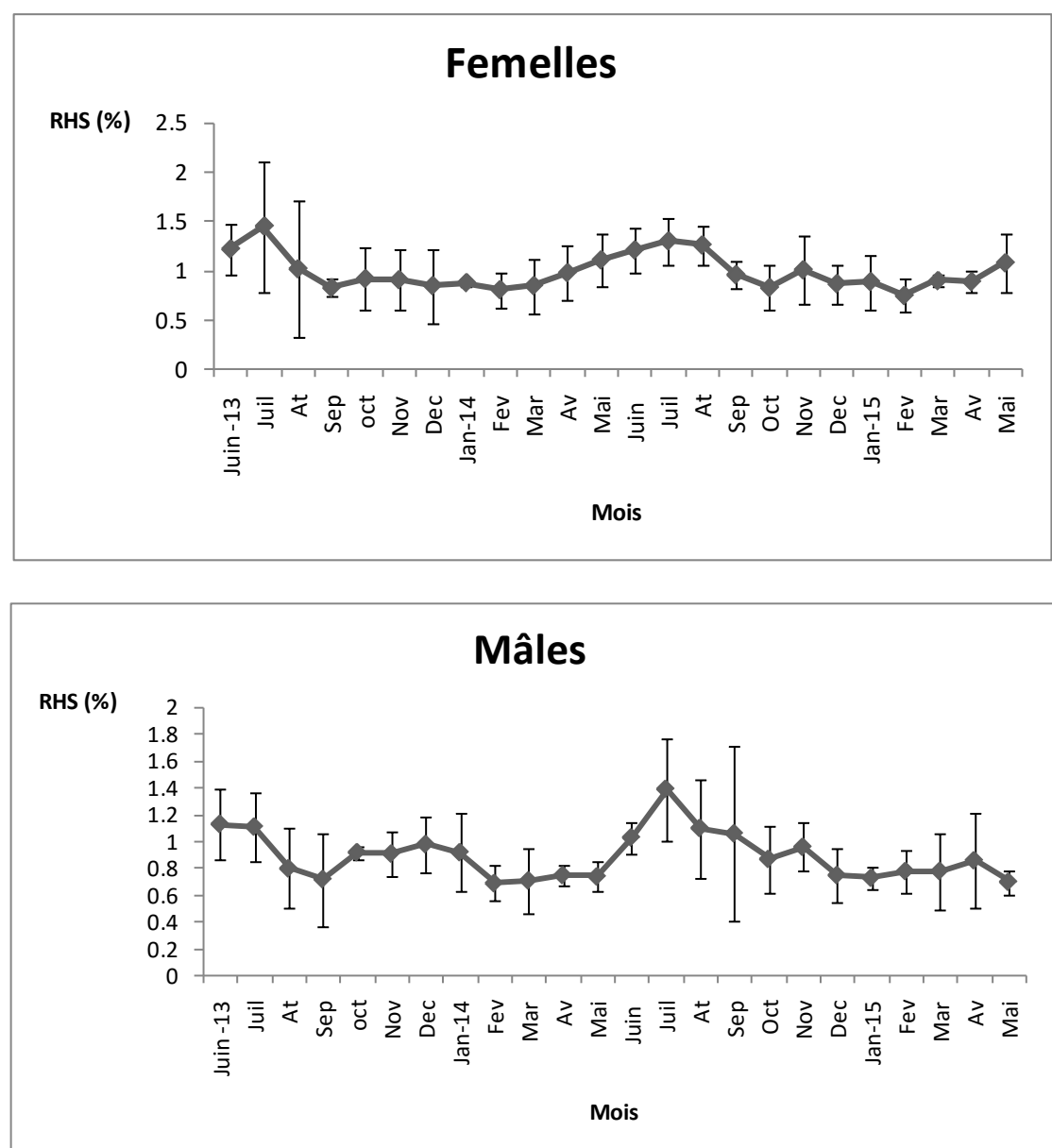

Figure 4 : Variations mensuelles du rapport hépato-somatique (RHS) des femelles et des mâles de Heterotis niloticus de la rivière Agnéby de juin 2013 à mai 2015.

\section{Facteur de condition}

Les courbes de variation mensuelle du facteur de condition ont une évolution similaire chez les deux sexes (Figure 5). Les valeurs maximales de $\mathrm{K}$ pour les femelles et les mâles sont observées aux mois d'août 2014 $(1,58 \pm 0,12$ et $1,36 \pm 0,33$ respectivement) et les valeurs minimales sont enregistrées en mai 2014 pour les femelles $(1,08 \pm 0,04)$ et en juillet 2013 pour les mâles $(0,9 \pm 0,02)$. 

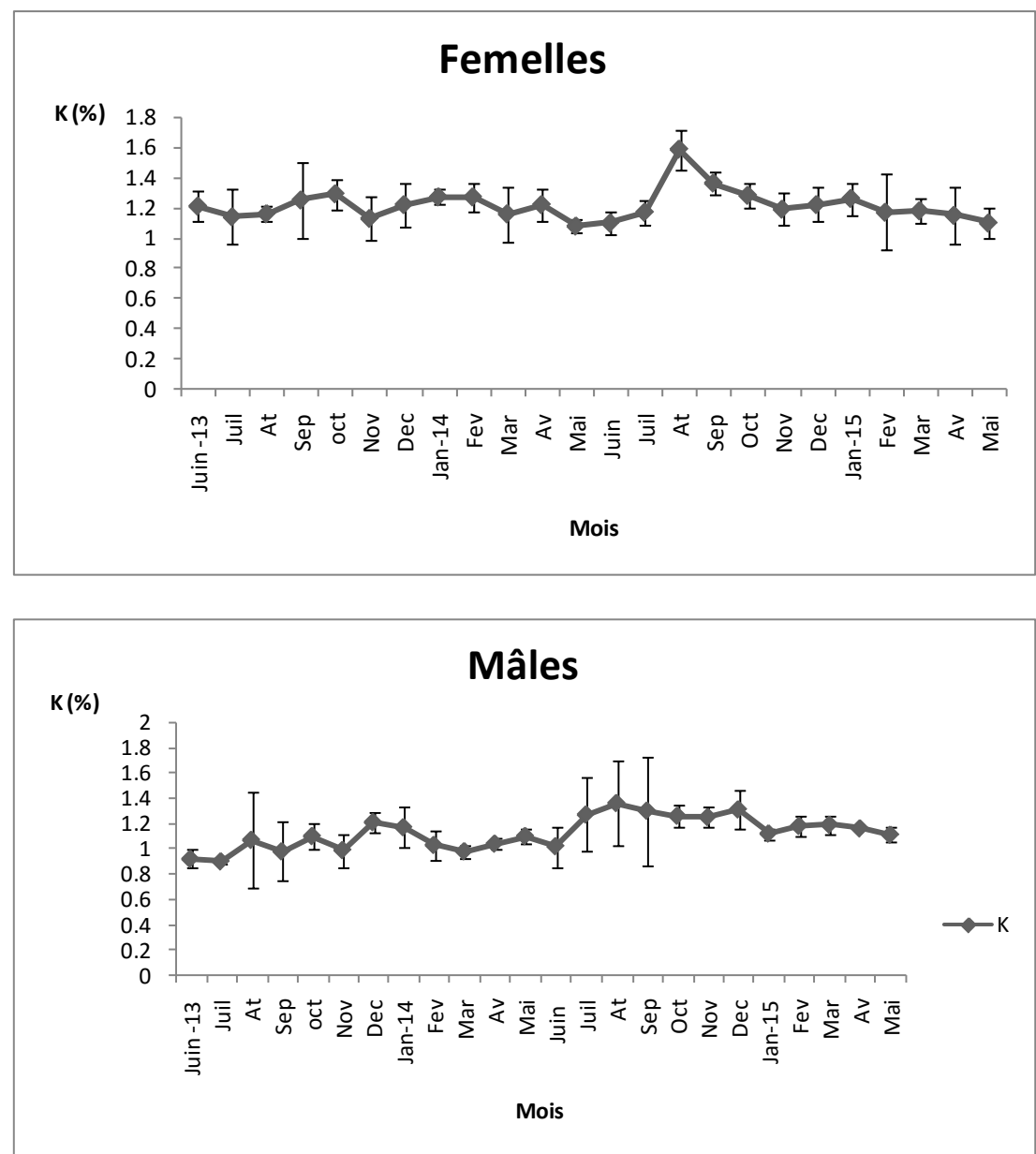

Figure 5: Variations mensuelles du facteur de condition (K) des femelles et des mâles de Heterotis niloticus de la rivière Agnéby de juin 2013 à mai 2015.

\section{Taille à la première maturité sexuelle}

La taille de première maturité sexuelle $\left(\mathrm{Ls}_{50}\right)$ est de 490,6 mm (LT = $540 \mathrm{~mm}$ ) chez les femelles et 436,3 $\mathrm{mm}(\mathrm{LT}=480 \mathrm{~mm})$ chez les mâles (Figure 6). Le test de $\mathrm{khi}^{2}$ ne montre pas de différence significative entre ces deux longueurs $\left(X^{2}=0,32\right)$.

La plus petite taille mature chez les femelles est de $330 \mathrm{~mm}$ de longueur standard $(\mathrm{LT}=370 \mathrm{~mm}$ ) et celle des mâles est de $300 \mathrm{~mm}$ de longueur standard $(\mathrm{LT}=340 \mathrm{~mm})$. 

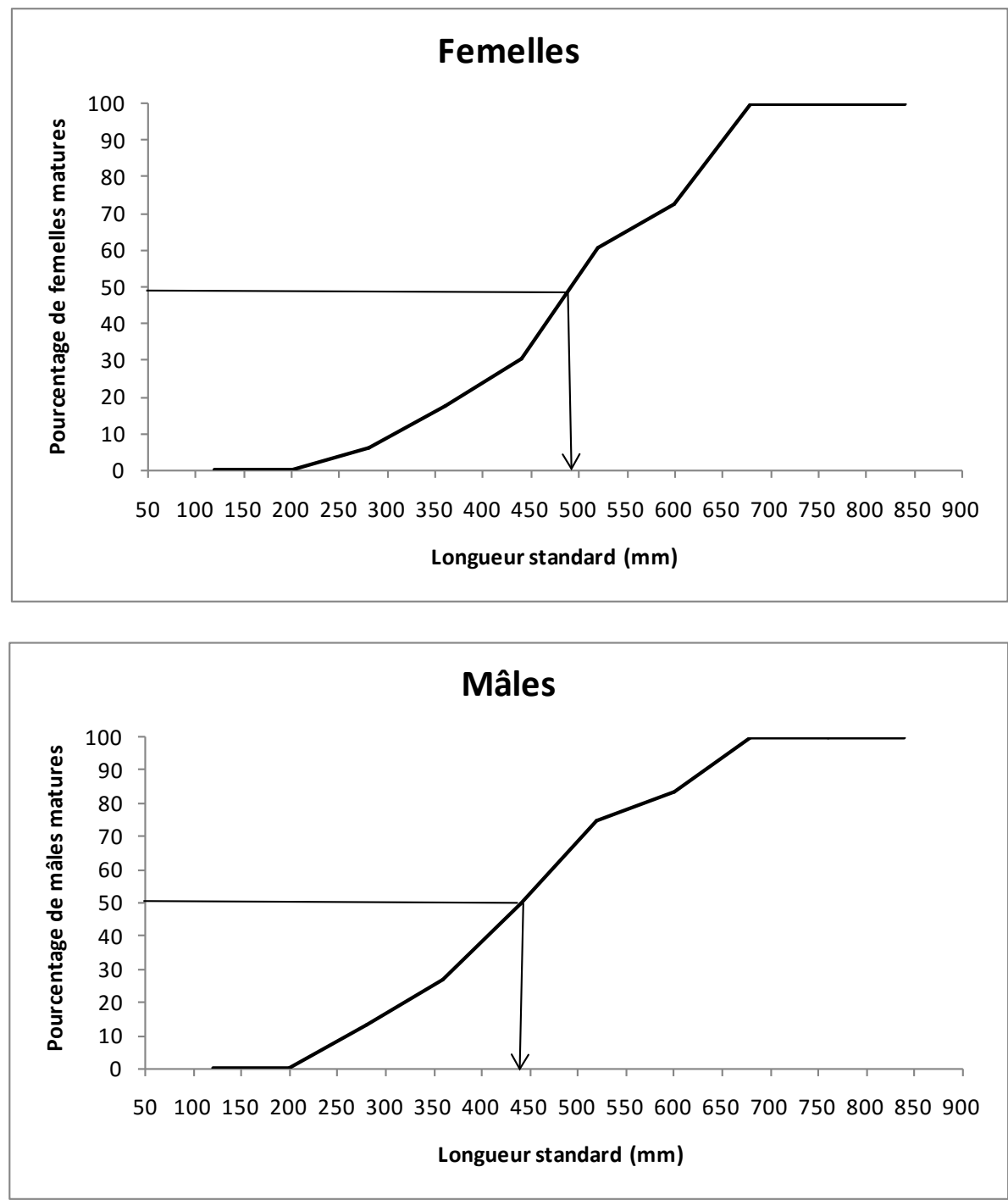

Figure 7: Courbes pour la détermination de la taille de première maturité sexuelle en fonction de la longueur standard $\left(\mathrm{Ls}_{50}\right)$ des femelles et mâles de Heterotis niloticus de la rivière Agnéby.

\section{Fécondité}

La fécondité absolue varie de 5424 à 25591 ovocytes avec une valeur moyenne de $12692 \pm 5596 \mathrm{chez}$ des femelles dont la longueur standard est comprise entre 595 et $780 \mathrm{~mm}$.

Concernant la fécondité relative, elle est comprise entre 2079 et 7216 ovocytes/kg de poids corporel avec valeur une moyenne de $3752 \pm 1491$ ovocytes/kg de poids corporel. 


\section{Discussion}

La sex-ratio observée pour l'ensemble des captures de Heterotis niloticus montre une prédominance des femelles $(1: 1,17)$ dans la rivière Agnéby. Excepté les mois de juin et juillet. La variation saisonnière révèle la prédominance des mâles pendant la saison pluvieuse et celle des femelles pendant la saison sèche. Selon Aka et al. (2004), la variation de la sex-ratio dépend de l'état physiologique des poissons. Généralement chez les téléostéens, les mâles sont prédominants en période de reproduction alors qu'en période de repos sexuel, ce sont les femelles qui sont prédominantes (Santos et al., 2007; Sylla et al, 2009; Djadji et al., 2013). En effet, le nombre des mâles augmente considérablement pendant la période de reproduction du fait que ces derniers se concentrent autour des femelles pour la reproduction (Chemmam-Abdelkader et al., 2002; Kraidy et al., 2014). Selon Konan et al. (2013), la prédominance des mâles durant certains mois pluvieux pourrait être due à la migration de certaines femelles vers des zones spécifiques qui aurait rendu plus difficile leur capture. En effet, $H$. niloticus pratique la garde parentale et les femelles pondent dans des zones de végétation. A partir du moment où les œufs sont pondus, l'un des géniteurs reste constamment à proximité du nid ou même se tient au milieu de celui-ci; par sa présence et ses mouvements, il empêche les débris, herbes et feuilles de se déposer sur les œufs, éloigne les prédateurs éventuels et contribue à renouveler l'eau au contact des alevins (Moreau, 1982). Cela pourrait expliquer le nombre élevé des mâles dans les prises des pêcheurs. Reizer (1964) , Micha (1973) et Moreau (1982) ont observé une sex-ratio équilibrée $(1: 1)$ pour ce poisson dans leur travaux respectifs.

Les valeurs de la taille de première maturité sexuelle obtenues dans la rivière Agnéby sont de 436,3 mm (LS) pour les mâles soit $480 \mathrm{~mm}$ (LT) et 490,6 mm (LS) pour les femelles soit 540 mm (LT). Dans la rivière Sô au Nigéria, les individus de cette même espèce de sexe confondu sont matures à $575 \mathrm{~mm}$ de longueur totale (Adite et al., 2006). La taille de première maturité observée dans l'Oubangui en République Démographique du Congo est de $400 \mathrm{~mm}$ de longueur totale (Micha, 1973). Moreau (1982) stipule que tous les individus de poids supérieur à $1 \mathrm{~kg}$ sont pubères. Les différences observées s'expliquent par le fait que la taille de maturité des individus dépend des facteurs biologiques et/ou écologiques du milieu (Wague et M’Bodj, 2002 ; Koné et al., 2011 ). Selon Lévêque et al. (2006), les poissons développent des stratégies en fonction du milieu en vue d'une meilleure adaptation et de tirer profit de leur écosystème. Les activités anthropiques telles que la pression de pêche et l'utilisation de certains engins de pêche expliqueraient les différences de taille de première maturité (Kraidy et $a l$. , 2014). 
Les variations mensuelles des rapports gonado-somatiques, hépatosomatiques et du facteur de condition indiquent que la reproduction de $H$. niloticus dans la rivière Agnéby a lieu principalement deux fois dans l'année. La première période de reproduction se déroule de juin à août et la seconde de novembre à décembre. Les courbes de variations mensuelles du rapport hépato-somatique et du rapport gonado-somatique évoluent dans le même sens selon les deux sexes. Cependant, le pic du RGS précède celui du RHS. Ceci indiquerait que $H$. niloticus est un poisson maigre c'est-à-dire que ses réserves énergétiques s’accumulent surtout au niveau du foie (Djadji et al., 2013). Dans ce cas, les réserves emmagasinées dans le foie sont utilisées au profit du développement des gonades; autrement dit au cours de la maturation des gonades le poisson puise ses réserves dans le foie et non dans les muscles (Chemmam-Abdelkader et al., 2002).

La fécondité absolue varie de 5424 à 25591 ovocytes pour des femelles de 595 à $780 \mathrm{~mm}$ de longueur standard. Quand à la fécondité relative, elle varie entre 2079 et 7216 ovocytes/Kg de poids corporel. Dans la rivière Sô au Nigéria, la fécondité absolue varie entre 2700 à 27500 ovocytes pour des femelles de 500 à $735 \mathrm{~mm}$ de longueur standard (Adite et al., 2006). Micha (1973) observe dans l'Oubangui une fécondité absolue variant entre 3572 et 15246 ovocytes pour des femelles de 560 à $820 \mathrm{~mm}$ de longueur totale. $H$. Niloticus peut être consideré comme une espèce n’appartennant pas aux poissons à très forte fécondité. En effet, les espèces à très forte fécondité produisent des millions d'œufs (Sylla et al, 2009; Djadji et al., 2013).

\section{Conclusion}

Cette étude montre que dans la rivière Agnéby, Heterotis niloticus a deux périodes de reproduction allant de juin à août et de novembre à décembre. La taille de première maturité sexuelle $\left(\mathrm{Ls}_{50}\right)$ est atteinte à une longueur standard de 436,3 chez le mâle et 490,6 mm chez la femelle. La fécondité absolue varie de 5424 à 25591 ovocytes avec une valeur moyenne de $12692 \pm 5596$.

\section{References:}

Adite A., Winemiller K. O., Fiogbe E. D. Population structure and reproduction of the African bonytongue Heterotis niloticus in the Sô Riverfloodplain system (West Africa): implications for management. Ecology of Freshwater Fish, 15: 30-39. 2006.

Aka Z., Koç H. T., Turan C. A study on the anchovy Engraulis encrasicolus, Linnaeus (1758) in Turkish Seas, Turkey. Pakistan Journal of Biological Sciences, 7 (7): 1121-1126. 2004. 
Bony K.Y. Biodiversité et écologie des mollusques Gasteropodes d'eau douce en milieu continental Ivoirien (Bassin de l’Agnéby, de la Mé et du Banco). Traits d'histoire de vie d'une espèce invasive Indoplanorbis exustus (Deshayes, 1834). Thèse de doctorat de l’Université Abobo Adjamé. 217p. 2007.

Chemmam-Abdelkader B., Kraiem M. M., El Abed, A. Période de ponte, sex-ratio et maturité sexuelle de Dentex maroccanus (Teleostei, Sparidae) des côtes Tunisiennes. Bulletin de l' Insitut National des Sciences et Technologies de la Mer de Salammbô, (29): 5-10. 2002.

Djadji E. L.G., Atsé B. C., Sylla S., Konan K. J., Kouassi N. J. Reproduction du Mugilidae Mugil cephalus Linné, 1758 dans deux complexes lagunaires (lagunes Ébrié et de Grand-Lahou) de la Côte d'Ivoire. International Journal of Biological and Chemical Sciences, 7(4): 1701-1716. 2013.

Diomandé D., Bony K. Y., Edia O. E., Konan K. F., Gourène,G. Diversité des Macroinvertébrés Benthiques de la Rivière Agnéby (Côte d’Ivoire; Afrique de l'Ouest). European Journal of Scientific Research, 35 (3) : 368377. 2009.

Girard G., Sircoulon J., Touchebeuf P. Aperçu sur les régimes hydrologiques. In : Le milieu naturel de la Côte d'Ivoire. ORSTOM. Paris 50:113-151. 1971.

Goula B. T. A., Kouadio Z. A., Kouakou K. E., N’Go, Y. A., N’Doumé, C., Savané, I. Simulation du comportement hydrologique du bassin versant de L’Agnéby, en Côte d'Ivoire. Revue Ivoirienne des Sciences et Technologies, 13 : 91 -113. 2009.

Gourène G., Teugels G.G. Manuel pratique d'identification des poissons du lac d’Ayamé (rivière Bia, Côte d'Ivoire). Archive scientifique, 14 (1): 41p. 1995.

Kao H.C., Chan T.Y., Yu H.P. Ovary development of the Deep-water Shrimp Aristaeomorpha Foliacea (Risso, 1826) (Crustacea: Decapoda: Aristeidae) from Taiwan. Zoological Studies, 38 (4): 373-378. 1999.

Koné N., Berté S., Kraidy A. L. B., Kouamelan E. P., Koné T. Biologie de la reproduction du Clupeidae Pellonula leonensis Boulenger, 1916 dans le lac de barrage de Kossou (Côte d'Ivoire). Journal of Applied Biosciences, 41 : 2797-2807. 2011.

Konan Y. A., Ouattara S., Koné T., Bamba M., Koné I. Caractéristiques de la reproduction de Thysochromis ansorgii (Pisces, Cichlidae) dans la forêt des marais Tanoé-Ehy (Côte d'Ivoire). Journal of Applied Biosciences, 71 : 5715-5727. 2013.

Kraidy A. B. L., Koné N., Berté S., Konan N. G., Yao S. S., Kouamelan E. $\mathrm{P}$. Pêche et paramètres de reproduction de Pellonula leonensis Boulenger 1916, dans le lac de Taabo (Fleuve Bandama, Côte d’Ivoire): implications 
pour une exploitation durable du stock. International Journal of Biological and Chemical Sciences, 8(1): 75-88. 2014.

Lévêque C., Paugy D., Duponchelle F. La reproduction. In Les Poissons des Eaux Continentales Africaines : Diversité, Ecologie, Utilisation par l'Homme, Lévêque C, Paugy D. (eds). Editions de l’IRD: Paris. 148-176. 2006.

Micha J. C. Etude des populations piscicoles de l’Ubangui et tentatives de sélection et d'adaptation de quelques espèces à l'étang de pisciculture. Centre Technique Forestier Tropical. Document Bibliothèque CIRAD. 110p. 1973.

Monentcham M. S. E. Alimentation et Nutrition des juvéniles de Heterotis niloticus (Arapaimidae, Teleostei). Premières estimations des besoins nutritionnels et valorisation des sous-produits végétaux. Thèse de Doctorat des Facultés Universitaires Notre Dame de la Paix Namur. 144p. 2009.

Moreau J. Exposé synoptique des données biologiques sur Heterotis niloticus (Cuvier, 1829). Synopsis FAO sur les Pêches, vol 131. 45p. 1982.

Moreau J., Moreau I. Etude du cycle annuel de la gamétogenèse chez Heterotis niloticus au lac Ivakoina (zone de Pangalanes) Madagascar. Revue d'Hydrobiologie Tropicale, 15 (3) : 271-280. 1982.

Offem B. O., Samsons Y. A., Omoniyi I. T. Trophic ecology of commercially important fishes in the cross river, Nigeria. Journal of Animal and Plant Sciences, 19(1): 37-44. 2009.

Parkinson D., Petit F., Perpinien G., Philippart J.C. Habitats de reproduction des poissons et processus géomorphologiques dans des rivières a fond caillouteux. Essai de synthèse et applications a quelques rivières du bassin de la meuse. Bulletin de la Société Geographique de Liège, 31-52. 1999.

Reizer C. Comportement et reproduction d'Heterotis niloticus en petits étangs. Revue Bois et Forêts des Tropiques, 95 : 49-60. 1964.

Santos J. N. S., Araùjo F. G., Silva M. A., Vasconcellos R. M. Sex ratio and sexual dimorphism of the anchovy Anchoa januaria (Actinopterygii, Engraulidae) in a tropical bay in south-eastern Brazil. Journal of Fish Biology, 71: 877-888. 2007.

Sylla S., Atsé B. C., Kouassi N. J. Stratégie de Reproduction du Carangidae Trachinotus teraia Cuvier, 1832 dans la lagune Ebrié (Côte d’Ivoire). Sciences et Nature, 6 (1) : 83 - 94. 2009.

Wague A., M'Bodj O.B. Etude de quelques aspects de la reproduction chez la sardinelle ronde Sardinella aurita (Valenciennes, 1847) pêchée le long des côtes mauritaniennes. Bulletin Scientifique de l'Institut Mauritanien de Recherches Océanographiques et des Pêches, 29: 13-18. 2002. 